This is an author produced version of a paper published in Experimental Neurology. This paper has been peer-reviewed but does not include the final publisher proof-corrections or journal pagination.

Citation for the published paper:

Thompson, Lachlan H and Andersson, Elin and Jensen, Josephine B and Barraud, Perrine and Guillemot, Francois and Parmar, Malin and Björklund, Anders

"Neurogenin2 identifies a transplantable dopamine neuron precursor in the developing ventral mesencephalon."

Exp Neurol. 2006 Mar;198(1):183-98.

http://dx.doi.org/10.1016/j.expneurol.2005.11.025.

Access to the published version may require journal subscription.

Published with permission from: Elsevier 


\section{Neurogenin2 identifies a transplantable dopamine neuron precursor in the developing ventral mesencephalon}

Lachlan H. Thompson ${ }^{1 *}$, Elin Andersson ${ }^{1} *$, Josephine B. Jensen ${ }^{1}$, Perrine Barraud ${ }^{1}$, Francois Guillemot ${ }^{2}$, Malin Parmar ${ }^{1}$ and Anders Björklund ${ }^{1}$

${ }^{1}$ Wallenberg Neuroscience Center, Department of Experimental Medical Science, and Lund Strategic Center for Stem Cell Biology and Cell Therapy, Lund University, BMC A11, SE22184 Lund, Sweden. ${ }^{2}$ National Institute for Medical Research, Division of Molecular Neurobiology, The Ridgeway Mill Hill, NW7 1AA UK.

*These authors contributed equally to this work.

Abbreviated title: Neurogenin2 identifies DA neuron precursors

Key words: Cell sorting, FACS, transplantation, Parkinson's disease

\section{Figures}

30 pages

Address for correspondence:

Anders Björklund

Wallenberg Neuroscience Center

Division of Neurobiology

Lund University

BMC A11

S-221 84 Lund, Sweden

Phone: +46 462220541

Fax: +46 462220559

Email: anders.bjorklund@med.lu.se

\section{Acknowledgements:}

We thank Ulla Jarl, Anneli Josefsson, Elsy Ling and Bengt Mattsson for technical assistance, Anna Fossum and Zhi Ma at the Lund Stem Cell Center for expert assistance in the FACS procedures, and Drs. H. Edlund, T. Edlund, A. Joyner, R. Lindahl, and M. Nakafuku for their kind gift of antibodies. This work was supported by grants from the Swedish Research Council and the EuroStemCell Integrated Program grant from the European Commission. The Lund Stem Cell Center is supported by a Center of Excellence Grant from the Swedish Foundation for Strategic Research (SSF). 
In neural transplantation studies there is an interest in identifying and isolating mesencephalic dopamine (mesDA) neuron precursors that have the capacity to differentiate into fully mature mesDA neurons after transplantation. We report here that in the developing ventral mesencephalon (VM) the proneural gene Neurogenin2 (Ngn2) is expressed exclusively in the part of the ventricular zone that gives rise to the migrating mesDA neuroblasts, but not in the differentiated mesDA neurons. From other studies we know that Ngn2 is involved in the generation of mesDA neurons and that the development of mesDA neurons is severely compromised in Ngn2-null mutant mice. We show here that cells isolated by FACS from the developing VM of Ngn2-GFP knock-in mice are capable of generating mesDA neurons, both in vitro and after transplantation to the striatum of neonatal rats. All mesDA neuron precursors, but not the serotonergic or GABAergic neuron precursors, are contained in the Ngn2-GFP-expressing population. Moreover, all glial cells were generated from cells contained in the GFP-negative cell fraction. The results show that surviving mesDA neurons in VM grafts are derived from early postmitotic, probably Nurr1-expressing precursors before they have acquired their fully differentiated neuronal phenotype. The Ngn2-GFP reporter construct used here thus provides a tool for the identification of mesDA neuron precursors in the VM and selective isolation of transplantable mesDA neuron precursors for transplantation. 


\section{INTRODUCTION}

Transplantation of dopamine (DA) neurons in Parkinson's disease (PD) has so far been based on the use of post-mitotic DA neuroblasts obtained from the developing ventral mesencephalon (VM). In the clinical setting this is a serious limitation since the postmitotic DA neuroblasts are non-expandable, and thus limited in number, which has made it necessary to use multiple fetal donors in order to obtain sufficient numbers of cells for transplantation in each PD patient $(6,38)$. The use of primary fetal tissue, moreover, is unsatisfactory since it is difficult to standardize, and the composition, quality and viability of this tissue material cannot be properly controlled. For further development of this approach, therefore, there is a need to develop techniques for identification and isolation of DA neuron precursors from the developing VM that are capable of differentiating into fully mature DA neurons after transplantation to the DA-denervated striatum.

Dopaminergic neurons make up defined sets of cells in the hypothalamus, olfactory bulb and retina; however, the bulk of the DA neurons are found in the VM $(10,20,22)$. These mesencephalic dopaminergic (mesDA) neurons have received particular attention due to their involvement in cognitive and motor processes. The substantia nigra (SN, cell group A9), is the area primarily degenerated in $\mathrm{PD}$, while the ventral tegmental area (VTA, group A10), has been implicated in affective disorders, drug-dependency and schizophrenia. During embryogenesis, the mesDA neurons are formed during a 3-4 day period in the mouse, starting around embryonic day (E) 11 and continuing until about E1415 (4, 17). Several developmental genes, such as Engrailed (En) 1 and 2, and Lmx1b, and cellular proteins such as aldehyde dehydrogenase-1 (Aldh1, also known as AHD-2), have been shown to be expressed in DA neuron precursors in the developing VM. However, the expression of these proteins is not restricted to the precursor pool, but is maintained also in the differentiated, post-mitotic mesDA neurons $(9,14,21,30,32,37)$. Here we report that 
the proneural gene product Neurogenin2 (Ngn2) within the VM is expressed exclusively in the part of the proliferating ventricular zone (VZ), which gives rise to the migrating mesDA neuron precursors, but not in the differentiated, tyrosine hydroxylase (TH)-expressing postmitotic mesDA neurons.

In parallel studies we (3) and others (18) have shown that Ngn2 is essential for the proper development of mesDA neurons and that the generation of both the SN and the VTA DA neuron systems are severely compromised in Ngn2 knock-out mice.

Using a transgenic mouse in which GFP has been knocked in to the Ngn2 locus, we show that these Ngn2-GFP expressing progenitors, when isolated from fetal VM by FACS, do indeed give rise to the mesDA neurons, but not to other neuronal types such as serotonergic, cholinergic and GABAergic neurons. Furthermore, we identify the cells from the developing VM that give rise to dopaminergic neurons in vivo following transplantation as post-mitotic, Nurr1+/TH- neuroblasts derived from the Ngn2-expressing progenitors within the ventricular zone.

\section{MATERIALS and METHODS}

Animals and tissue preparation: All procedures were conducted in accordance with rules set by the Ethical Committee for the use of laboratory animals at Lund university. Timed pregnant (day of vaginal plug = E0.5) mice were generated by crossing wild type $(w t)$ NMRI females with either wt or +/- Ngn2-GFP CD1/129 males. Following lethal exposure to $\mathrm{CO}_{2}$ the embryos were removed and either taken for FACS analysis or immersion fixed overnight in $4 \%$ paraformaldehyde (PFA) in phosphate buffered saline (PBS; $0.1 \mathrm{M}$ ) at $8^{\circ} \mathrm{C}$. Early stage (E10.5 - E13.5) or late stage (E15.5-17.5) fixed embryos were cryoprotected in $30 \%$ or $25 \%$ sucrose respectively and cryo-sectioned at a thickness of $12-16$ $\mu \mathrm{m}$. 
Fluorescence Activated Cell Sorting (FACS): E12.5 Ngn2-GFP or wt VM tissue was dissected as previously described $(11,25)$ and cell suspensions were prepared by mechanical dissociation in PBS without $\mathrm{Ca}^{2+}$ or $\mathrm{Mg}^{2+}\left(\mathrm{PBS}^{-\mathrm{Ca} 2+/ \mathrm{Mg} 2+}\right.$; Gibco) with $1 \mathrm{mM}$ EDTA and $0.5 \%$ bovine serum albumin at a concentration of approximately $3.0 \times 10^{6}$ cells/ml. To identify and eliminate dead cells, 7-aminoactionomycin-D (7AAD; Sigma) was included at $10 \mu \mathrm{l} / \mathrm{ml}$. VM cells from Ngn2-GFP heterozygous embryos were sorted based on fluorescence intensity. GFP ${ }^{\text {high }}, \mathrm{GFP}^{\text {low }}$ and $\mathrm{GFP}^{\text {neg }}$ fractions were collected for in vitro analysis and $\mathrm{GFP}^{\text {pos }}\left(\mathrm{GFP}^{\text {high }}+\mathrm{GFP}^{\text {low }}\right)$ and $\mathrm{GFP}^{\text {neg }}$ fractions were collected for transplantation (as illustrated in Fig. 5R). Gate settings for GFP ${ }^{\text {neg }}$ were determined using wt tissue, while gate settings for $\mathrm{GFP}^{\text {high }}$ and $\mathrm{GFP}^{\text {low }}$ were chosen arbitrarily. The purity was confirmed through reanalysis as $>95 \%$ for the $\mathrm{GFP}^{\text {neg }}$ and $\mathrm{GFP}^{\text {high }}$ fractions and > 85\% for GFP ${ }^{\text {low }}$. An 'unsorted' cell preparation was generated for transplantation by passing wt cells through the FACS and gating for live cells. All FACS procedures were carried out using a FACS Diva flow cytometer (Becton-Dickson) equipped with $488 \mathrm{~nm}$ argon and 633 nm Helium-Neon lasers.

Transplantation procedure: Unsorted, $\mathrm{GFP}^{\text {neg }}$ or $\mathrm{GFP}^{\mathrm{pos}}$ cells were resuspended in $\mathrm{HBSS}^{-}$ $\mathrm{Ca} 2+/ \mathrm{Mg} 2+$ (Gibco) at $0.5 \times 10^{5}$ cells/ $\mu$ l. Under deep hypothermic anaesthesia, $1 \mu \mathrm{l}$ of cell suspension was delivered over 2 minutes to the striatum of postnatal day 3 Sprague Dawely rats, as previously described (25). Injection co-ordinates (mm) were 0.7 anterior and 1.9 lateral to bregma, and 2.9 below the dural surface. Four weeks after transplantation animals received lethal doses of pentobarbitone and were trans-cardially perfused with $0.9 \%$ saline followed by 4\% PFA. The brains were post-fixed for 2 hours, cryoprotected in $25 \%$ sucrose and sectioned on a freezing microtome (30 $\mu \mathrm{m}$, coronal sections). 
In vitro differentiation: Following sorting into $\mathrm{GFP}^{\text {high }}, \mathrm{GFP}^{\text {low }}$ and $\mathrm{GFP}^{\text {neg }}$ fractions, cells were re-suspended at $2.5 \times 10^{5}$ cells/ $\mu$ l in DMEM/F-12 (Gibco) supplemented with a defined hormone/salt mixture (27). The suspensions were plated at a density of $1.2 \times 10^{5}$ cells/cm² $\left(2 \times 10^{5}\right.$ cells/well) in poly-l-lysine coated 4-well chamber slides. Following two days of culture in the presence of $10 \mathrm{ng} / \mathrm{ml}$ bFGF and $20 \mathrm{ng} / \mathrm{ml}$ EGF (both R\&D systems), the growth factors were withdrawn and the medium changed to include $1 \%$ foetal bovine serum (for differentiating conditions) for a further 5 days. Bromo-deoxyuridine (BrdU, 0.2 $\mu \mathrm{M}$; Sigma) was included for the first $48 \mathrm{~h}$ in some cultures. Parallel cultures were maintained under differentiating conditions for 7 days. In all cultures the medium was replenished at 3-day intervals. The cultures were fixed with ice-cold 4\% PFA for 15 min at room temperature.

Immunohistochemistry: The procedure for immunostaining cell cultures or tissue sections was carried out as previously described (12). Briefly, sections and culture slides were preincubated for 1 hour in blocking solution containing 5\% normal serum and $0.25 \%$ triton-X (Ameresco) in KPBS. Primary antibodies diluted in blocking solution were applied overnight at room temperature. After rinses, biotinylated or fluorophore-conjugated secondary antibodies (diluted 1:200 in blocking solution) were applied for 2 hours at room temperature. Biotinylated secondary antibodies were followed either by incubation with streptavidin-horseradish peroxidase complex (ABC elite kit, Vectastain) for 1-1.5 hours and subsequent exposure to di-amino-benzydine (DAB, $0.5 \mathrm{mg} / \mathrm{ml}$; Sigma) or by fluorophore-conjugated streptavidin for 1 hour (Cy2-, Cy3- and Cy5-streptavidin, 1:200, Jackson Labs). Where appropriate, endogenous peroxidase activity was quenched for 15 min with $3 \% \mathrm{H}_{2} \mathrm{O}_{2}$ prior to pre-incubation. Pretreatment for detection of BrdU was as 
previously described (8). Primary antibodies and dilution factors were as follows: rabbit anti-5HT (1:10000; Incstar), rabbit anti-Aldh1 (1:500; gift from R.Lindahl), mouse antiBIII-tubulin (1:300; Sigma), rabbit anti-ChAT (1:1000; Chemicon), rabbit anti-En1/2 (1:1000; gift from A.Joyner), rabbit anti-GABA (1:250; Sigma), rabbit anti-GFAP (1:1000; Dako), chicken anti-GFP (1:5000; Chemicon), rabbit anti-Isl1/2 (1:500; gift from H. and T. Edlund), rabbit anti-Isl 1 (1:100; Abcam), rabbit anti-Ki67 (1:100; Dianova), rat antiM2M6 (1:50; Hybridoma bank), mouse anti-Nestin (1:10; BD Biosciences), rabbit antiNgn2 (1:1000; gift from M. Nakafuku), rabbit anti-Nurr1 (1:1000; Santa Cruz), mouse antiParvalbumin (1:2000; Sigma), rabbit anti-Pbx1/2/3 (1:400; Santa Cruz), rabbit anti-TH (1:1000; Pelfreeze). Secondary antibodies used were: biotinylated swine-anti-rabbit (1:200, DAKO), goat-anti-rabbit or horse-anti-mouse; FITC- or Cy2-conjugated donkeyanti-chicken or donkey-anti-rabbit; Cy3-conjugated donkey-anti-rat or donkey anti-rabbit; Cy5-conjugated donkey-anti-mouse (all 1:200, Jackson Laboratories).

In situ hybridization histochemistry: In situ hybridization was performed as previously described (36) using a digoxigenin labeled cRNA probe for Ngn2 (for probe specifications see Gradwohl et al., 1996).

Quantification and statistics: For quantification of the immunostained cell cultures, total numbers of TH- or 5HT-positive cells were counted in triplicate wells from each of the GFP fractions. The percentage of BrdU labeled cells was quantified by counting BrdUpositive and total cell numbers (based on phase-contrast) in random visual fields. This was repeated in 4 wells from each GFP fraction and 400 - 650 cells were counted per well. Where cell aggregates had formed the cell number was estimated based on the size of the aggregate. The difference in $\mathrm{TH}+, 5 \mathrm{HT}+$ or BrdU+ cell numbers was compared using a 
one-way ANOVA with Fisher's correction. The number of TH+ or 5HT+ cells contained within the grafts ( $\mathrm{n}=4$ per group) was counted in every $12^{\text {th }}$ tissue section and the total number was estimated using the Abercrombie (1) formula. The difference in $\mathrm{TH}+$ and $5 \mathrm{HT}+$ cell numbers between groups was determined using one-way ANOVA with Fisher's correction.

\section{RESULTS}

Ngn2 is expressed in the VM proliferative ventricular zone during DA neurogenesis

The mesDA neuron precursors are known to be generated in a restricted area of the VM ventricular zone, close to the midline. As they leave the cell cycle, they migrate ventrally along radial glia toward the ventral surface and differentiate into TH-positive neurons within the mantle zone $(17,29,34)$. Depending on detection method and age determination of the embryos, the first TH immunoreactive cells in mouse appear in the medial aspect of the VM around E11 $(13,17)$. With our method of dating (vaginal plug = E0.5) and antibody (rabbit polyclonal, PelFreeze) the mesDA neurons expressing TH could be detected starting from E11.5 and in all embryos at subsequent stages. Cells expressing the Ngn2 protein could be detected by immunohistochemistry in cells residing in the proliferative VZ as early as E10.5, i.e., at a time point shortly before the first THexpressing dopaminergic neurons start to appear (data not shown). At E11.5, when the first TH-expressing neurons appear in the mantle zone (Fig. 1D), Ngn2-expressing cells are found in the VZ immediately overlying the newly formed TH-expressing DA neurons (Fig. 1B,J). Adjacent sections stained for Ngn2 and the cell cycle protein Ki67, showed that the Ngn2-expressing cells are contained within the dorso-ventral boundary of the Ki67-positive proliferative domain (Fig. 1J,K), and in situ hybridization histochemistry of Ngn2 mRNA indicated that $\mathrm{Ngn} 2$ is expressed at highest levels at the ventral edge of this zone (Fig 1A). 
The vast majority of cells in this layer stain positively for the intermediate filament protein Nestin, which is commonly used as a marker for dividing progenitors in the developing CNS. Confocal analysis of Ngn2/Nestin double-stained sections show that the Ngn2expressing cells were also Nestin-positive (not shown).

In adjacent sections stained for Ngn2 and the early DA neuron precursor marker Aldh1, we found that the area of Ngn2 expression matches well with that of Aldh1 (Fig. 1B,E). However, while Aldh1 is expressed throughout both the ventricular and the mantle zone, including the TH-positive post-mitotic DA neurons, the Ngn2-expressing cells are confined to the precursors residing in the VZ. Most of the Ngn2-expressing cells are located within the Aldh1-positive domain, and the caudal boundary of Aldh1 expression also marks the caudal limit of Ngn2-expressing cells spanning the midline. Notably, however, at this developmental stage the band of Ngn2-expressing cells extends laterally, outside the Aldh1-expressing area, about half-way to the sulcus that marks the lateral border of the VM region (marked by arrow heads in Fig. 1). Cells expressing the more mature DA neuron markers Nurr1, En1/2 and TH are all located ventral to, but not overlapping with, the Ngn2-positive cells and the Ki67-positive domain (Fig. 1D,F,H). The Pbx homeodomain proteins are also expressed in the post-mitotic VM neurons within the mantle zone (Fig.1G) in a pattern similar to that of Nurr1 and TH. At this stage of development (E11.5) En1/2 is expressed at low levels also within the VZ, as well as in cells extending laterally, outside the Aldh1-positive DA neuron domain (Fig. 1H,M). Cells expressing Islet (Isl), which represent the precursors of the cholinergic motor neurons in the oculomotor nucleus $(2,37)$ are located in the intermediate zone of this lateral subsection of the VM, lateral to the Aldh1-positive DA neuron domain (Fig. 1I,N).

Analysis of Ngn2 expression at later stages of development showed that localization of Ngn2 expressing cells is spatially and temporally well correlated with the generation of 
DA neurons in the VM. At E12.5, Ngn2-expressing cells remained restricted to a narrow domain of the VZ spanning the midline, dorsal to the TH-positive DA neurons (Fig 2A-C). Similar to E11.5, the cells expressing the more mature DA neuron markers Nurr1, En1/2, Pbx and TH are all located ventral to the Ngn2-positive cells, and show no overlap (Fig. 2D-F). At E13.5, Ngn2 expression shows a clear rostro-caudal gradient: while some Ngn2expressing cells can still be detected rostrally in the VZ, most of the Ngn2-positive cells are now confined to the caudal part of the DA neuron domain (Fig. 2G-L). This is consistent with the pattern of DA neurogenesis described by Bayer et al (1995) where the germinal source of DA neurons is gradually shifted caudally during later stages of DA neurogenesis (E13-E14). The caudal shift of Ngn2 expression becomes even more evident at E15.5. At this stage, Ngn2-expressing cells can no longer be detected in the rostral part of the DA neuron domain (Fig. 2N,O), whereas some Ngn2-expressing cells are still detectable in the VZ in the caudal part (Fig. 2Q,R). At this level the TH-positive cells are clustered close to the midline (Fig 2P), consistent with the view that this part of the germinal neuroepithelium gives rise to DA neurons within the VTA (4). At E17.5, when no more mesDA neurons are generated, Ngn2 expression is no longer detectable within the VM and the DA neuron domain (not shown).

Ngn2 is specific for the DA neuron domain of the ventral mid/hindbrain region and is excluded from the area generating serotonergic neurons

In order to determine whether Ngn2 expression is specific to the part of the vz generating mesDA neurons, or whether it is also expressed by serotonergic neuron precursors, we investigated Ngn2 expression in sagittal sections and compared it to the position of the dopaminergic and serotonergic neurons. As seen in Fig. 3A, the THexpressing dopaminergic neurons are located rostral to the mid/hindbrain border (MHB; 
marked by dashed line), and the 5HT-expressing serotonergic cell group (Fig. 3D) is localized just caudal to the MHB. In adjacent sections the distribution of the Ngn2-positive precursors coincided with the $\mathrm{TH}$ expression domain, ending caudally precisely at the MHB, and were conspicuously absent from the rostral hindbrain region where the serotonergic neurons are formed (cf. Fig. 3B,C). As previously described (15, 33), Ngn2 expression extends rostrally into the developing diencephalon where it is expressed outside the zone of dopaminergic neurogenesis. Mash1, another bHLH transcription factor of the proneural family, displays an expression pattern complementary to Ngn2 in the forebrain and has recently been shown to be necessary for the development of brainstem serotonergic neurons (26). The Mash1-expressing cells were distributed both caudal to the MHB, corresponding to the location of the newly-formed 5HT-positive neurons (Fig. 3E,F), as well as rostral to the MHB, overlapping with the Ngn2 expression domain.

\section{Isolation of Ngn2 expressing cells by FACS}

In order to substantiate the apparent involvement of Ngn2 in mesDA neurogeneis, based on the expression data, we made use of a Ngn2-GFP knock-in mouse where the gene coding for Green Fluorescent Protein (GFP) is inserted into the Ngn2 locus. In the heterozygous Ngn2-GFP +/- mice, GFP is expressed in discrete regions of the developing cortex, diencephalon and lower brainstem-spinal cord, consistent with the previously described expression of $\operatorname{Ngn} 2(15,33)$, as well as the VM (Fig. 4A-C). In the VM, GFP is expressed within the $\mathrm{VZ}$ of the DA neuron domain, in a pattern closely resembling that of the Ngn2-expressing precursors (Fig.4D-G). Closer inspection of Ngn2/GFP doublestained sections showed that GFP is expressed not only in the Ngn2-positive cells in the proliferative zone, but also in cells with the morphology of migrating neuroblasts within the intermediate zone, immediately ventral to the Ngn2 expression domain (Fig. 4H-K). 
The GFP expression showed a marked dorso-ventral gradient, with the highest expression ventrally, in the intermediate zone (Fig. 4D,M). In the Ngn2-GFP +/- mice, Nurr1/GFP double-stained sections showed that most of these high GFP-expressing cells also express the Nurr1 protein and that virtually all Nurr1-positive cells in the intermediate zone were strongly GFP expressing (Fig. 4M-P). In TH/GFP double stained sections, TH-positive cells located in the dorsal aspect of the mantle zone were seen to express low levels of the GFP protein. This extended pattern of GFP expression suggests that the GFP protein, due to its relatively long half-life, accumulates over time in the Ngn2-expressing cells and remains detectable for some time, probably some 12-24 hours, after the endogenous Ngn2 production has been switched off.

At E12.5 GFP-expressing cells, albeit in lower number, occurred also in the lateral part of the VM, outside the DA neuron domain (Fig. 4D,M,Q). Since they do not appear to express either Nurr1 (Fig 4.N,O) or TH (Fig. 4F,G) they are unlikely to represent DA neuron precursors. In this lateral domain we also see Isl-positive cells, i.e., the precursors of the cholinergic motor neurons in the oculomotor nucleus (Fig. 4R,S). At E11.5 Ngn2expressing cells are observed in a lateral position in the VM (Fig.1J) and at this stage the Isl-positive cells were weakly GFP-expressing suggesting that they are derived from Ngn2expressing cells. However, one day later, at E12.5 (i.e. the stage used in the cell sorting experiment) Ngn2 was no longer expressed in this lateral position (Fig. 2B), and GFP was no longer detectable in the Isl-positive cells (Fig. 4Q-T).

The native fluorescence of the GFP protein allowed us to isolate the GFP-positive $\left(\mathrm{GFP}^{\text {pos }}\right)$ cell population by FACS from the VM of E12.5 Ngn2-GFP +/- fetuses. The microscopy data indicate that the FACS-sorted GFP ${ }^{\text {pos }}$ cell population (which constituted approx. $60 \%$ of all VM cells; see Fig. 5R) will contain not only the Ngn2-containing precursors in the $\mathrm{VZ}$ but also their immediate progeny, i.e. the post-mitotic migratory 
neuroblasts within the intermediate zone, as well as precursors from the lateral part of the VM not destined to generate DA neurons. The dissected tissue included the whole VM and extended caudal to the MHB, including also part of the hindbrain serotonin neuron domain.

The ability of the sorted GFP ${ }^{\text {pos }}$ and GFP ${ }^{\text {neg }}$ cell fractions to generate DA neurons was studied in vitro after plating on poly-l-lysine coated chamber slides and culturing for 7 days. As indicated in Fig. 5R, the GFP ${ }^{\text {pos }}$ cells were separated into two fractions: GFP ${ }^{\text {high }}$, containing primarily the GFP-expressing migratory post-mitotic neuroblasts in the intermediate zone, and $G F P^{l o w}$, containing the Ngn2-positive precursors in the proliferative VZ that expressed lower levels of GFP. The latter cell fraction is also expected to include some differentiated TH-positive cells from the mantle zone that retained the GFP protein (see above). In all three cell cultures, $\mathrm{GFP}^{\text {high }}, \mathrm{GFP}^{\text {low }}$ and $\mathrm{GFP}^{\text {neg }}$, the vast majority, 70$80 \%$, of the cells were $\beta$ III-tubulin positive with neuron-like morphology. TH-positive neurons were found exclusively in the cultures generated from the $\mathrm{GFP}^{\text {pos }}$ cell fractions, and their number was 3-fold higher in the GFP ${ }^{\text {high }}$ compared to the GFP ${ }^{\text {low }}$ cultures (Fig. 5A-E). 5HT-positive neurons, by contrast, were found in the GFP ${ }^{\text {neg }}$ cell cultures, but not in the ones generated from the GFP ${ }^{\text {high }}$ cell fraction (Fig. 5C). Some few 5HT-positive cells were present also in the GFP ${ }^{\text {low }}$ cultures, which may reflect the spill-over of non-GFP expressing cells into the GFP ${ }^{l o w}$ fraction due to the difficulty of distinguishing lowexpressing GFP cells from background. Cells incorporating BrdU (present during the first two days of culture) were found in all three types of culture, but the number of BrdUlabeled cells was 3-4-fold higher in the GFP neg and $\mathrm{GFP}^{\text {low }}$ cultures compared to the cultures generated from the GFP ${ }^{\text {high }}$ fraction (Fig. 5C,G,K,O). Although BrdU-positive cells were observed in the $\mathrm{GFP}^{l o w}$ and GFP ${ }^{\text {high }}$ cultures, only single $\mathrm{TH}+/ \mathrm{BrdU}+$ doublelabeled cells could be detected, and the vast majority of the TH-positive neurons were 
BrdU-negative (Fig. 5J-M,N-Q) indicating the they had exited the cell cycle at the time of plating.

In vivo differentiation of the FACS-sorted cells after transplantation to the striatum

The GFP ${ }^{\text {pos }}$ (high and low combined) and GFP ${ }^{\text {neg }}$ cell fractions, defined as indicated in Fig. 5R, as well as VM cells passed though the FACS, gated for live cells but without sorting ("unsorted" cells), were transplanted to the striatum of neonatal rats. The grafts were analysed by immunohistochemistry 4 weeks after transplantation using the mousespecific antibody M2M6 for general visualization of neurons and glia in combination with the cell-specific markers, TH, 5HT, GABA, Parvalbumin, Isl, ChAT and GFAP.

In all three groups the M2M6-positive cells occurred clustered at the injection site, and some of the cells had migrated for some distance into the host striatal parenchyma (Fig. 6A,E,I). Note that glial cells are stained particularly intensely with the M2M6 antibody and are present in the unsorted (Fig. 6A) and GFP ${ }^{\text {neg }}$ (Fig. 6E) derived grafts but are absent from the GFP ${ }^{\text {pos }}$ derived graft (Fig. 6I). The unsorted VM cells (Fig. 6A-D) gave rise to both neurons and glia, with extensive M2M6-positive neurites in striatal fiber bundles (Fig. 6P) and extending into the striatum and the overlying cortex (Fig. 6M). Abundant M2M6positive fibers were also seen to project through the corpus callosum into the contralateral hemisphere, and along the internal capsule towards the globus pallidus and the entopeduncular nucleus (Fig. 6M-Q). Double-stained sections revealed the presence of $\mathrm{TH}+$, 5HT+, GABA+/Parvalbumin+ and Isl+/ChAT+ neurons, as well as large number of neurons expressing none of these markers. Glial cells with the morphology of astrocytes had migrated in large numbers into the host striatum.

The GFP ${ }^{\text {pos }}$ and $\mathrm{GFP}^{\text {neg }}$ cell fractions gave rise to neuron-rich grafts with the same general appearance as the ones generated from the unsorted cells, but they differed in their 
composition: $\mathrm{TH}+$ neurons were found only in the grafts derived from the GFP ${ }^{\text {pos }}$ cell fraction (Fig. 6F,J). By contrast, 5HT+ neurons (Fig. 6G,K), GABA+/Parvalbumin+ neurons (Fig. 7I-P) and Isl+/ChAT+ neurons (Fig. 7A-H) occurred almost exclusively in the GFP ${ }^{\text {neg }}$ cell grafts. These data indicate that the mesDA neuron precursors indeed were confined to the GFP ${ }^{\text {pos }}$ cell population, and that the serotonergic, GABAergic and cholinergic neurons were derived from cells negative for this marker (note that the Isl+ cells were weakly GFP-positive at E11.5 but GFP-negative at E12.5, i.e. at the stage used for FACS sorting). The TH+ cells formed in the GFP ${ }^{\text {pos }}$ cell grafts had the morphology of fully mature mesDA neurons, with elaborated dendritic and axonal processes, similar to the ones seen in the unsorted grafts, and in grafts of primary fetal VM tissue. The number of $\mathrm{TH}+$ neurons averaged $941 \pm 342$ in the unsorted grafts, $1258 \pm 466$ in the GFP ${ }^{\text {pos }}$ grafts, and less than $20 \pm 18$ in the GFP ${ }^{\text {neg }}$ grafts. The yield of surviving, differentiated mesDA neurons, as a percentage of all cells transplanted, was about $1.9 \%$ in unsorted cell grafts and about $2.5 \%$ in the $\mathrm{GFP}^{\text {pos }}$ grafts. Both types of grafts contained large numbers of neurons not staining for either TH, 5HT, GABA or ChAT. Extensive M2M6-positive fiber outgrowth, similar to that observed in the grafts derived from unsorted cells, could be traced for long distances in striatum and cortex, and along the internal capsule towards globus pallidus and interpeduncular nucleus. This fiber outgrowth pattern was similar in $\mathrm{GFP}^{\text {pos }}$ and $\mathrm{GFP}^{\text {neg }}$ cell grafts.

The GFP ${ }^{p o s}$ and $\mathrm{GFP}^{\text {neg }}$ cell grafts differed also with respect to the glial content: while the $\mathrm{GFP}^{\text {neg }}$ cell grafts contained large numbers of both neurons and glia (Fig. 8A-C), the GFP ${ }^{p o s}$ cell grafts (Fig. 8D-F) were conspicuously devoid of glial cells. Consistent with this observation, M2M6/GFAP double-stained sections revealed abundant GFAP-positive astrocytes in the GFP ${ }^{\text {neg }}$ cell grafts but few or no such cells in the grafts derived from the $\mathrm{GFP}^{\text {pos }}$ cells (Fig. 8G-I). 


\section{DISCUSSION}

In the developing VM, Ngn2 expression is restricted to a population of cells located in the germinal zone overlying the newly-formed mesDA neurons. The first Ngn2-positive cells were detected at E10.5, i.e. slightly earlier than when the first TH expressing mesDA neurons started to appear, and were most prominent at the time-points associated with peak production of mesDA neurons. At later stages Ngn2 expression declined in a rostral-tocaudal gradient, much in the same way as has been reported for the mesDA neurogenesis (4). Ngn2 ceased to be expressed in this region at E15.5-17.5 when no more mesDA neurons are born.

Compared to other known developmental genes and proteins present in mesDA neuron precursors, or in post-mitotic DA neuroblasts, such as En1/2, Lmx1b, Aldh1, Pitx3 or Nurr1, Ngn2 appears to be unique in that it is confined to the precursor pool, and not expressed in the TH-expressing DA neurons. In the developing cortex Ngn2-expressing cells have been shown to incorporate BrdU, suggesting that at least in this area Ngn2 is expressed in actively dividing cells (15). In the VM the Ngn2-expressing cells were confined to the proliferative VZ, with the highest expression levels ventrally. Since these ventrally located Ngn2-expressing cells fail to show any labeling after injection of BrdU (Guillemot, unpublished data) they appear to be post-mitotic, suggesting that Ngn2 is expressed at highest levels in VM precursors at the time of cell cycle exit.

The mesDA neurons are generated within a narrow, midline-spanning medial sector of the VM. This DA neuron domain is well delineated by the expression of Aldh1, which is expressed both in DA neuron precursors in the VZ and in the post-mitotic DA neurons in the mantle zone (37). The Ngn2-positive precursors are largely confined to this sector, but 
at early stages of DA neurogenesis (E11.5) the Ngn2-positive precursors extend also laterally, outside the DA neuron domain, about half-way to the sulcus that marks the lateral margin of the VM area. Since no Nurr1- or TH-positive cells are generated in this area, these lateral Ngn2-expressing VM precursors are likely to give rise to non-DA neurons or other cell types.

\section{Generation of mesDA neurons from Ngn2-expressing VM precursors}

The Ngn2-GFP knock-in mouse allowed us to isolate the Ngn2-expressing cells from fetal VM by FACS. The advantage of this transgenic construct is that the GFP gene is inserted into the Ngn2 locus, which ensures that GFP transcription occurs only in cells where the Ngn2 gene is active. However, due to the relatively long half-life of the GFP protein it will be retained in the Ngn2-expressing cells for some time, probably 12-24 hours, after the Ngn2 gene is switched off. This allows, moreover, the cellular level of GFP to accumulate over time. The fact that the GFP protein is retained in the cells for some time after the endogenous Ngn2 production has ceased thus allows for a short-term fate mapping of the Ngn2 cell progeny within the VM. Indeed, at E12.5 (the stage used for FACS) the highest GFP levels were found in the post-mitotic, Nurr1-positive neuroblasts in the intermediate zone, which thus represent the progeny of the Ngn2-positive precursors in the overlying germinal zone. Virtually all Nurr1-positive cells expressed the GFP marker, indicating that all DA neurons within the VM derive from the Ngn2-positive precursors. Notably, in Ngn2-GFP +/+ mice of the same age (which represent functional Ngn2 knockouts) the GFP-expressing cells fail to adopt a Nurr1-positive phenotype and this is reflected also as a profound (approximately 90\%) reduction in TH-positive cell numbers at E13.5 - E15.5 (3) 
The Ngn2-positive precursors themselves expressed lower levels of the reporter. In the in vitro analysis, therefore, we studied the GFP $\mathrm{Gigh}^{\text {and }} \mathrm{GFP}^{\text {low }}$ fractions separately. THpositive neurons were generated exclusively from cells contained in the two GFP-positive cell fractions. However, they were 3-fold more numerous in the cultures generated from the $\mathrm{GFP}^{\text {high }}$ fraction and in both types of cultures the TH-positive cells, with few exceptions, had not incorporated the BrdU label. These data indicate that the surviving TH-positive neurons were generated from a portion of the GFP-labeled cells that had exited the cell cycle at the time of plating, and that this subpopulation was more abundant among the cells expressing the highest levels of GFP protein. Based on these observations we suggest that it is at or after cell cycle exit that the Ngn2-expressing VM precursors become fully committed to a mesDA neuron fate and are sufficiently specified to maintain this phenotype when removed from the developing VM. At this time-point Ngn2 is downregulated and the cell moves out from the proliferative VZ into the underlying intermediate zone. Soon thereafter, Nurr1 starts to be expressed in the post-mitotic neuroblasts. Since Nurr1 is known to play a critical role in the differentiation of DA neurons in the VM (28, 39,40 ) it seems likely that the ability of mesDA neuron precursors to generate TH-positive neurons is critically dependent on the expression of Nurr1. Consistent with this idea our data indicate that Ngn2-positive precursors have to mature into a Nurr1-positive DA neuroblast in order to be able to differentiate into a TH-positive mesDA neuron in vitro under the conditions used here, or in vivo after transplantation to the striatum. In support, (31) have reported that all TH-positive neurons present in fetal VM transplants are generated from cells that have exited the cell. The fully differentiated TH-positive mesDA neurons are likely to reside in the $\mathrm{GFP}^{\text {neg }}$ cell fraction. Very few TH-positive neurons, however, were obtained from GFP ${ }^{\text {neg }}$ cells either in vitro or in vivo. This suggests that the survival of the sorted mesDA neurons is very poor once they have become fully 
differentiated, which is consistent with previous observations $(7,11)$ that the yield of surviving mesDA neurons from grafted fetal VM tissue declines sharply once DA neurogenesis has ended. From the present data it thus seems that the VM precursors that are capable of generating surviving mesDA neurons after transplantation are derived from a narrow window of DA neuron development, i.e. from early post-mitotic, probably Nurr1expressing DA neuroblasts that have just exited the cell cycle, but have not yet acquired the fully differentiated, TH-expressing phenotype. This restricted time-window (probably some 12-18 hours of development) may explain why the overall yield of surviving TH-positive neurons in VM transplants is low, only about $2 \%$ (of all cells grafted) in the unsorted fraction used here, and in the range of $1-3 \%$ in grafts of VM cell suspensions prepared without cell sorting $(19,24)$.

Based on the finding that the DA progenitors are exclusively located within the GFP fraction, representing around $60 \%$ of VM cells, one might expect an enrichment in DA neurons of around 1.6-fold when comparing numbers in grafts of unsorted and GFP ${ }^{\text {pos }}$ cells. We observed, however, only a small and non-significant increase in DA neuron numbers in GFP-positive grafts (2.5\% of cells grafted) compared with the unsorted grafts (1.9\%) and this was not statistically significant. Considering the lack of glial cells in $\mathrm{GFP}^{\text {pos }}$ grafts and the reported role of VM glia in DA neuron development and survival (16), one may well speculate that poor survival of DA neurons in grafts lacking VM glia might counter any enrichment in the progenitor pool in terms of the overall number of surviving DA neurons. The Ngn2-GFP mouse represents a valuable tool for further studies in this area.

Fate commitment of Ngn2-expressing VM precursors

The sorted E12.5 VM cells survived well after transplantation and differentiated into fully mature neurons and glia. Tyrosine hydroxylase-positive neurons developed exclusively 
from cells contained in the GFP ${ }^{p o s}$ cell fraction, while 5HT+, GABA+/Parvalbumin+ and Isl+/ChAT+ neurons were derived almost exclusively from the GFP ${ }^{\text {neg }}$ cells. Glial cells, moreover, developed in large numbers in the GFP ${ }^{\text {neg }}$ cell grafts, but not in the GFP ${ }^{\text {pos }}$ ones. As in other regions of the CNS (5), the role of $N g n 2$ in the VM is likely to be two-fold: (i) to induce a neuronal phenotype, coupled to exit from the cell cycle, and (ii) to assist in the regional specification of the newly-formed neuroblasts toward a specific neuronal phenotype. The virtual absence of glial cells in the GFP ${ }^{p o s}$ cell grafts is in line with the neurogenic property of the neurogenin proteins, which are known to induce neurogenesis in uncommitted progenitors, and at the same time inhibit their differentiation into glial cells $(5,23,35)$. The GFP ${ }^{p o s}$ cell fraction, however, was clearly heterogenous. Large numbers of neurons present in the GFP ${ }^{\text {pos }}$ cell grafts did not stain for the $\mathrm{TH}$ marker, suggesting that the Ngn2-expressing progenitors are able to generate also other, non-dopaminergic, neuronal phenotypes. The transmitter phenotype of this non-dopaminergic progeny is unclear. Their extensive axonal outgrowth patterns, as observed in M2M6-immunostained sections, suggest that they may represent neurons with long axonal projections. Some of these are likely to derive from the Ngn2-expressing VM cells located lateral to the DA neuron domain. Alternatively, it seems possible that not all cells in the GFP ${ }^{\text {pos }}$ cell fraction have reached the stage of fate commitment where they are able to differentiate cellautonomously in the host striatum, i.e. in an environment that is likely to lack critical instructive cues for DA neuron differentiation. In the absence of such external cues some of the specified, but not yet fully determined, precursors in this fraction may fail to undergo full DA neuron differentiation. It is interesting to compare the present results with those previously obtained by Sawamoto et al (2001). In this study, VM cells expressing GFP under the control of the Nestin enhancer were obtained by FACS from Nestin-GFP transgenic mice and rats. The Nestin-positive cells clearly represent a broader progenitor 
cell population than the one used here, but it will contain also the Ngn2-expressing VM precursors. In the Sawamoto et al (2001) study, TH-positive neurons were generated from Nestin-GFP-positive cell fraction, but at a lower frequency than from the Ngn2-GFPpositive cells used here (1.25\% compared to $2.5 \%$ of all grafted cells). This suggests that early post-mitotic DA neuroblasts, i.e. the cells that can generate mesDA neurons after transplantation, are contained also in the Nestin-GFP-positive cell fraction, but at a relatively lower frequency than in the Ngn2-GFP-positive cell fraction.

In conclusion, the results show that Ngn2 is expressed in a subpopulation of neuronal progenitors in the VM ventricular zone. When isolated by FACS from the VM of Ngn2GFP knock-in mice, the Ngn2-GFP-expressing progenitor population was shown to contain all cells with the capacity to differentiate into mesDA neurons, both in vitro and in vivo after transplantation to the neonatal striatum, while other neuronal subtypes, as well as glial cells, were generated from the Ngn2-GFP-negative fraction. The results show that the transplantable mesDA neuron precursors are derived from the Ngn2 expressing cells within the VM ventricular zone. Our data indicate that the Ngn2-GFP expressing cells that are capable of surviving and generating mesDA neurons after transplantation are the early post-mitotic, Nurr1-expressing DA neuroblasts that have just exited the cell cycle and have not yet started to express the TH enzyme. This has important implication for studies aimed at generating and isolating transplantable DA neuron generating precursors from embryonic and neural stem cells. 


\section{FIGURE LEGENDS}

Figure 1 - Ngn2 expression in the developing VM. $A-N$ : Coronal sections through the VM of E11.5 mice. Ngn2 mRNA (A) and protein (B) expression are localized within the medial part of the VM extending from the midline approximately halfway to the lateral sulcus (marked by arrowheads). The Ngn2-expressing cells are confined to the proliferative vz (J), defined by dividing cells expressing the cell-cycle marker, Ki67 (C, K). MesDA neurons expressing $\mathrm{TH}$ are located within the same medio-lateral expression domain as Ngn2, but are positioned more ventrally, in the mantle zone (D). Other markers found within the mesDA neuron domain include: Aldh1 (E, L), which is expressed in dividing precursors as well as post-mitotic DA neurons; Nurr1 (F), Pbx (G) and En1/2 (H, M) which are expressed in post-mitotic DA neurons; and Isl (I, N), which is expressed in the precursors of the cholinergic neurons of the oculomotor nucleus. Scale bars: A-I, $1 \mathrm{~mm}$; J$\mathrm{N}, 400 \mu \mathrm{m}$. 
Figure 2 - Ngn2 is expressed in a temporal and spatial pattern that matches the development of mesDA neurons. A-Q: At E12.5 (A-F), Ngn2 expressing cells are located immediately dorsal to, but not overlapping with, cells expressing markers for more mature DA neurons, including TH (A-C), En1/2 (D), Nurr1 (E) and Pbx (F). In C, Ngn2 is stained in red and TH in blue. At E13.5 (G-I), very few Ngn2+ cells are seen in the rostral aspects of the VM $(\mathrm{H}, \mathrm{I})$ but persist in more caudal regions $(\mathrm{K}, \mathrm{L})$, consistent with the rostral to caudal temporal pattern of mesDA neurogenesis, such that the more rostral cells are born before the more caudal ones (note the greater number of TH cells in G compared to J). By E15.5 (M-R), when mesDA neurogenesis is near completion (M, P), very few Ngn2+ cells are seen either rostrally $(\mathrm{N}, \mathrm{O})$ or caudally $(\mathrm{Q}, \mathrm{R})$ in the $\mathrm{VM}$. The double-labeling in $C$ was captured by confocal microscopy. Scale bars: A, $1 \mathrm{~mm}$ (in B, D-F); C, $0.2 \mathrm{~mm}$; G, $1 \mathrm{~mm}$ (in H, J, K, M, N, P and Q); I, $500 \mu \mathrm{m}$ (in L, O and R).

Figure 3 - Ngn2 expression is excluded from the developing hindbrain. $A-F$ : Immunohistochemical staining of adjacent sagittal sections from the brainstem of E12.5 mice. The boxed area (inset in A and D) indicates the region represented in the photographic images. Both TH (A) and Ngn2 (C) are expressed ventrally in a similar pattern extending caudally from the diencephalon throughout the mesencephalic flexure and ending sharply at the mid-hindbrain border (MHB). The newly formed 5HT+ (serotonergic) neurons are located in the hindbrain, caudal to the MHB (D, E). Mash1+ cells are distributed throughout the hindbrain and the mesencephalon and can be found within the proliferative vz directly dorsal to the 5HT+ cells (F). Scale bars: A-F, 1 mm. 
Figure 4 - The Ngn2-GFP reporter mouse at E12.5. Native GFP fluorescence (B) can be seen in a number of discrete locations within the developing Ngn2-GFP mouse brain (A, phase contrast; C, merge of $\mathrm{A}$ and $\mathrm{B}$ ). Dashed lines in panel $C$ indicate the VM region taken when dissecting, which extends slightly caudally beyond the mid-hindbrain border. Triple-labeled confocal images of coronal sections through the VM showing the expression patterns of GFP (D), Ngn2 (E), TH (F) and their overlap (G). Many of the cells expressing both Ngn2 and GFP have the morphology of migrating neuroblasts (H-J). A close inspection of the GFP expression domain (K) shows that GFP is expressed at low levels in the Ngn2+ cells within the VZ and continues to be expressed at high levels in the more mature, Ngn2- cells in the intermediate zone (delineated by dashed lines), and at low or undetectable levels in the TH+ cells within the mantle zone. The cells with high GFP expression in the intermediate zone are predominately Ngn2- and TH-, although a few scattered $\mathrm{TH}+$ cells can be found in this region $(\mathrm{K}, \mathrm{L})$. The expression pattern of Nurr1 overlaps extensively with the GFP+ cells in the intermediate zone (M-P). Isl expression can be found just ventral to the more laterally located GFP+ cells of the intermediate zone (QS). At this stage (E12.5), the Isl+ cells themselves do not express detectable levels of GFP (T). Abbreviations: BS, brainstem; iz, intermediate zone; mz, mantle zone; Tel, telencephalon; Thal, thalamus; VM, ventral mesencephalon; vz, ventricular zone. Scale bars: $200 \mu \mathrm{m}$ in D-G, M-O and Q-S; $50 \mu \mathrm{m}$ in K-L, P and T; $5 \mu \mathrm{m}$ in H-J.

Figure 5 - In vitro analysis of E12.5 VM cells from Ngn2-GFP +/- mice following FACS. Single cell suspensions of E12.5 VM tissue contain both viable and dead cells, which can be separated based on 7AAD exclusion (R, left panel). The viable cells (boxed area) were further analysed for GFP expression and gated into GFP ${ }^{\text {neg }}, G F P^{\text {low }}$ and $G F P^{\text {high }}$ fractions according to GFP intensity ( $\mathrm{R}$, middle panel) relative to control tissue from wild 
type VM. The same data represented as a histogram (R, right panel) shows the relative quantity of cells comprising these three fractions, with the distribution of cells from wild type VM (grey) superimposed on the Ngn2-GFP VM curve (green). In the middle and right panels, the limit of signal intensity for GFP ${ }^{\text {neg }}$ cells (as determined by FACS of wild type VM tissue) is indicated by the vertical red line.

When cells are differentiated in culture directly after FACS, the GFP reporter can no longer be detected from $\mathrm{GFP}^{\text {neg }}$ or $\mathrm{GFP}^{\mathrm{pos}}$ fractions, either via native fluorescence or when amplified immunohistochemically (not shown). Approximately equal numbers of neurons are derived from the different GFP fractions - panels $A$ and $D$ show immunohistochemistry for $\beta$ III-tubulin in representative fields from $\mathrm{GFP}^{\text {neg }}$ and $\mathrm{GFP}^{\text {high }}$ fractions respectively. The types of neurons in the different GFP fractions vary markedly however, with $5 \mathrm{HT}+$ cells segregating predominately in the GFP ${ }^{\text {neg }}$ fraction (B) and $\mathrm{TH}+$ cells in the $\mathrm{GFP}^{\text {high }}$ fraction (E). The bar chart in panel $C$ gives a quantitative description of the distribution of $\mathrm{TH}+$ (green bars) and 5HT+ (dark red bars) cells across the GFP ${ }^{\text {neg }}$, $\mathrm{GFP}^{\text {low }}$ and $\mathrm{GFP}^{\text {high }}$ fractions. Relative to either the GFP ${ }^{\text {low }}$ of $\mathrm{GFP}^{\text {high }}$ groups, the $\mathrm{GFP}^{\text {neg }}$ group contained significantly more $5 \mathrm{HT}+$ cells and significantly less $\mathrm{TH}+$ cells $(\mathrm{p}<0.05$, $\mathrm{n}=3$ ). The light red bars in panel $C$ represent the percentage of cells incorporating BrdU after a $48 \mathrm{~h}$ pulse added at the time of plating. Cells from all three GFP fractions formed small cellular aggregates, as shown in phase contrast images ( F, J and N). Many cells from the $\mathrm{GFP}^{\text {neg }}(\mathrm{G})$ and $\mathrm{GFP}^{\text {low }}(\mathrm{K})$ fractions incorporated BrdU, while significantly fewer cells from the $\mathrm{GFP}^{\text {high }}$ fraction $(\mathrm{O})$ were labelled (light red bars in $\mathrm{C} ; \mathrm{p}<0.01, \mathrm{n}=4$ ). There were also significantly more BrdU+ cells in the $\mathrm{GFP}^{\text {low }}$ fraction compared to the $\mathrm{GFP}^{\text {neg }}$ fraction (p $<0.01, n=4)$. Upon differentiation, cells from the $\mathrm{GFP}^{\text {low }}(\mathrm{L})$ and $\mathrm{GFP}^{\text {high }}(\mathrm{P})$ but not $\mathrm{GFP}^{\text {neg }}(\mathrm{H})$ fraction gave rise to $\mathrm{TH}+$ neurons (green bars in $\mathrm{C}$ ). With single exceptions (arrowheads in panels $\mathrm{J}-\mathrm{M}$ ), the $\mathrm{TH}+$ cells did not incorporate BrdU and were thus most 
likely post-mitotic at the time of plating. Abbreviations: 7AAD, 7-aminoactionomycin-D; SSC, side-scatter. Scale for 7AAD or GFP and SSC represent fluorescence intensity and internal cell complexity respectively, given in arbitrary units. Scale bars: A, $100 \mu \mathrm{m}$ (bar in A is relevant for A-D; bar in F is relevant for F-Q). Statistics: One-way ANOVA with Fishers correction for multiple comparisons.

Figure 6 - Grafts of sorted E12.5 VM cells from Ngn2-GFP +/- mice. Grafts of the unsorted (A-D), GFP ${ }^{\text {neg }}(\mathrm{E}-\mathrm{H})$ or GFP $\mathrm{P}^{\mathrm{pos}}(\mathrm{I}-\mathrm{L}) \mathrm{VM}$ cell fractions were identified 4 weeks later by immunohistochemistry using the mouse specific M2M6 marker (red). Note that M2M6 antibody stains glial cells more intensely than neurons. Glial cells are abundant both in the graft core and scattered in the surrounding host striatum in the unsorted (A) and $\mathrm{GFP}^{\text {neg }}(\mathrm{E})$ grafts, but are absent from the GFP ${ }^{\text {pos }}$ graft (I). Confocal microscopy of triple immunohistochemical labelling shows that grafts derived from unsorted cells contain both $\mathrm{TH}+(\mathrm{B})$ and $5 \mathrm{HT}+(\mathrm{C})$ cells; the $\mathrm{GFP}^{\text {neg }}$ grafts contained very few $\mathrm{TH}+$ cells $(\mathrm{F})$ and many $5 \mathrm{HT}+$ cells $(\mathrm{G})$, the $\mathrm{GFP}^{\mathrm{pos}}$ grafts contained many TH+ cells $(\mathrm{J})$ and very few $5 \mathrm{HT}+$ cells $(\mathrm{K})$. The quantitative analysis $(\mathrm{O})$ showed that the $\mathrm{GFP}^{\mathrm{pos}}$ grafts contained significantly more $\mathrm{TH}+$ cells and significantly less 5HT+ cells than the GFP ${ }^{\text {neg }}$ grafts $(\mathrm{p}<0.01$ in each case, $n=4)$. D, H and $L$ show high power images of M2M6+ neuronal processes not staining for either TH or $5 \mathrm{HT}$ emanating from unsorted (D), GFP ${ }^{\text {neg }}(\mathrm{H})$ and $\mathrm{GFP}^{\text {pos }}$ grafts (L). (Asterixes mark the location of these images in the low power images in A, E and I for spatial reference). These M2M6+ processes could be traced over long distances in cortex (M), corpus callosum (N), along the striatal fibre bundles (P; M2M6 fibres appear white, striatal fibre bundles are grey), and in the globus pallidus (Q). Scale bars: $200 \mu \mathrm{m}$ in A-C, E-G and I-K; $50 \mu \mathrm{m}$ in D, H and L. Statistics: One-way ANOVA with Fishers correction for multiple comparisons. 
Figure 7 - Isl+ and GABAergic neurons in grafts of sorted E12.5 VM cells from Ngn2GFP +/- mice. Confocal microscopy of triple immunostaining for either M2M6, Isl and ChAT (A-H) or M2M6, GABA and parvalbumin (I-P) in $\mathrm{GFP}^{\text {neg }}$ and GFP ${ }^{\text {pos }}$ grafts. A comparison of Isl immunohistochemistry in grafts derived from $\mathrm{GFP}^{\text {neg }}(\mathrm{B})$ or $\mathrm{GFP}^{\mathrm{pos}}(\mathrm{F})$ cells shows that Isl+ cells are present in both types, but that they are more prevalent in the GFP $^{\text {neg }}$ grafts. Many of the Isl+ cells in both graft types co-express ChAT (arrowheads in B-D and F-H). GABA+ cells are also much more abundant in grafts from GFP ${ }^{\text {neg }}$ cells (J) than in GFP ${ }^{\text {pos }}$ grafts $(\mathrm{N})$. Many of these GABA+ cells co-express parvalbumin (arrow in JL) and some are GABA+ /Parvalbumin- (arrowhead in J-L). Note the abundance of M2M6+ glial cells in the GFP ${ }^{\text {neg }}$ grafts (A and I), which are notably absent in the GFP ${ }^{\text {pos }}$ grafts (E and M). The M2M6 antigen is distributed on the cell surface of most neurons and is absent from the cytoplasm such that the M2M6-positive neurons appear as 'pockets' of M2M6-negative spaces within the graft cores, representing the cytoplasm of neuronal cell bodies. Scale bar: in A $50 \mu \mathrm{m}$ for all panels.

Figure 8 - Glia in grafts of sorted E12.5 VM cells from Ngn2-GFP +/- mice. Grafts derived from $\mathrm{GFP}^{\text {neg }}$ cells contain a mixture of neurons and glia as identified by immunohistochemistry for M2M6 (A-C). Many of the glial cells migrate some distance away from the graft core and are distributed as single cells or small cell clusters around the core (A). Some of these cells co-express GFAP (G-I, magnification of lower boxed area in A). The boxed areas in A, illustrated at higher magnification in B, C and G-I show the presence of GFAP+ cells within the GFP ${ }^{\text {neg }}$ graft core. Grafts derived from GFP ${ }^{\text {pos }}$ cells do not contain any glial cells that can be identified based on morphology or immunohistochemistry for GFAP (D-F). The boxed area in D at higher magnification 
illustrates the lack of cells within the GFP ${ }^{\text {pos }}$ graft core with the M2M6 staining intensity and morphology typical of glia (E) and also a lack of GFAP+ cells (F). Scale bars: A and D, $400 \mu \mathrm{m}$; B-C and E-F, $200 \mu \mathrm{m}$; G-I, $50 \mu \mathrm{m}$. 


\section{REFERENCES}

1. Abercrombie, M. 1946. Estimation of nuclear population from microtome sections. The anatomical record 94: 239-247.

2. Agarwala, S., and Ragsdale, C. W. 2002. A role for midbrain arcs in nucleogenesis. Development 129: 5779-5788.

3. Andersson, E., Jensen, J. B., Parmar, M., Guillemot, F., and Bjorklund, A. Development of the mesencephalic dopaminergic neuron system is compromised in the absence of Neurogenin2. Submitted.

4. Bayer, S. A., Wills, K. V., Triarhou, L. C., and Ghetti, B. 1995. Time of neuron origin and gradients of neurogenesis in midbrain dopaminergic neurons in the mouse. Exp Brain Res 105: 191-199.

5. Bertrand, N., Castro, D. S., and Guillemot, F. 2002. Proneural genes and the specification of neural cell types. Nat Rev Neurosci 3: 517-530.

6. Bjorklund, A., and Lindvall, O. 2000. Cell replacement therapies for central nervous system disorders. Nat Neurosci 3: 537-544.

7. Brundin, P., Barbin, G., Strecker, R. E., Isacson, O., Prochiantz, A., and Bjorklund, A. 1988. Survival and function of dissociated rat dopamine neurones grafted at different developmental stages or after being cultured in vitro. Brain Res 467: 233-243.

8. Caldwell, M. A., Garcion, E., terBorg, M. G., He, X., and Svendsen, C. N. 2004. Heparin stabilizes FGF-2 and modulates striatal precursor cell behavior in response to EGF. Exp Neurol 188: 408-420.

9. Chung, S., Hedlund, E., Hwang, M., Kim, D. W., Shin, B. S., Hwang, D. Y., Jung Kang, U., Isacson, O., and Kim, K. S. 2005. The homeodomain transcription factor Pitx3 facilitates differentiation of mouse embryonic stem cells into AHD2-expressing dopaminergic neurons. Mol Cell Neurosci 28: 241-252.

10. Dahlstrom, A., and Fuxe, K. 1964. Localization of monoamines in the lower brain stem. Experientia 20: 398-399.

11. Dunnett, S. B., and Björklund, A. 1992. Staging and dissection of rat embryos. In Neural Transplantation. A Practical Approach. (S. B. Dunnnet, and A. Björklund, Eds.), pp. 1-18. Oxford University Press, Oxford.

12. Englund, U., Fricker-Gates, R. A., Lundberg, C., Bjorklund, A., and Wictorin, K. 2002. Transplantation of human neural progenitor cells into the neonatal rat brain: extensive migration and differentiation with long-distance axonal projections. Exp Neurol 173: 1-21.

13. Foster, G. A., Schultzberg, M., Kokfelt, T., Goldstein, M., Hemmings, H. C., Jr., Ouimet, C. C., Walaas, S. I., and Greengard, P. 1988. Ontogeny of the dopamine and cyclic adenosine-3':5'-monophosphate-regulated phosphoprotein (DARPP-32) in the pre- and postnatal mouse central nervous system. Int J Dev Neurosci 6: 367-386.

14. Goridis, C., and Rohrer, H. 2002. Specification of catecholaminergic and serotonergic neurons. Nat Rev Neurosci 3: 531-541.

15. Gradwohl, G., Fode, C., and Guillemot, F. 1996. Restricted expression of a novel murine atonal-related bHLH protein in undifferentiated neural precursors. Dev Biol 180: 227-241.

16. Hall, A. C., Mira, H., Wagner, J., and Arenas, E. 2003. Region-specific effects of glia on neuronal induction and differentiation with a focus on dopaminergic neurons. Glia 43: 47-51. 
17. Kawano, H., Ohyama, K., Kawamura, K., and Nagatsu, I. 1995. Migration of dopaminergic neurons in the embryonic mesencephalon of mice. Brain Res Dev Brain Res 86: 101-113.

18. Kele, J., Simplicio, N., Ferri, A. L. M., Mira, H., Guillemot, F., Arenas, E., and Ang, S-L. Ngn2 is required for the development of ventral midbrain dopaminergic neurons. Submitted.

19. Kirik, D., Winkler, C., and Bjorklund, A. 2001. Growth and functional efficacy of intrastriatal nigral transplants depend on the extent of nigrostriatal degeneration. J Neurosci 21: 2889-2896.

20. Lindvall, O., Bjorklund, A., and Skagerberg, G. 1984. Selective histochemical demonstration of dopamine terminal systems in rat di- and telencephalon: new evidence for dopaminergic innervation of hypothalamic neurosecretory nuclei. Brain Res 306: 19-30.

21. McCaffery, P., and Drager, U. C. 1994. High levels of a retinoic acidgenerating dehydrogenase in the meso-telencephalic dopamine system. Proc Natl Acad Sci U S A 91: 7772-7776.

22. Nelson, E. L., Liang, C. L., Sinton, C. M., and German, D. C. 1996. Midbrain dopaminergic neurons in the mouse: computer-assisted mapping. J Comp Neurol 369: 361-371.

23. Nieto, M., Schuurmans, C., Britz, O., and Guillemot, F. 2001. Neural bHLH genes control the neuronal versus glial fate decision in cortical progenitors. Neuron 29: 401-413.

24. Nikkhah, G., Cunningham, M. G., Jodicke, A., Knappe, U., and Bjorklund, A. 1994. Improved graft survival and striatal reinnervation by microtransplantation of fetal nigral cell suspensions in the rat Parkinson model. Brain Res 633: 133-143.

25. Nikkhah, G., Winkler, C., Rödter, A., and Samii, M. 2000. Microtransplantation of nigral dopamine neurons: a "step by step" recipe. In Neuromethods: Cell and Tissue Transplantation in the CNS (S. B. Dunnett, A. A. Boulton, and G. B. Baker, Eds.), pp. 207-231. The Human Press, Totowa.

26. Pattyn, A., Simplicio, N., van Doorninck, J. H., Goridis, C., Guillemot, F., and Brunet, J. F. 2004. Ascl1/Mash1 is required for the development of central serotonergic neurons. Nat Neurosci 7: 589-595.

27. Reynolds, B. A., and Weiss, S. 1992. Generation of neurons and astrocytes from isolated cells of the adult mammalian central nervous system. Science 255: $1707-1710$.

28. Saucedo-Cardenas, O., Quintana-Hau, J. D., Le, W. D., Smidt, M. P., Cox, J. J., De Mayo, F., Burbach, J. P., and Conneely, O. M. 1998. Nurr1 is essential for the induction of the dopaminergic phenotype and the survival of ventral mesencephalic late dopaminergic precursor neurons. Proc Natl Acad Sci U S A 95: 4013-4018.

29. Shults, C. W., Hashimoto, R., Brady, R. M., and Gage, F. H. 1990. Dopaminergic cells align along radial glia in the developing mesencephalon of the rat. Neuroscience 38: 427-436.

30. Simon, H. H., Saueressig, H., Wurst, W., Goulding, M. D., and O'Leary, D. D. 2001. Fate of midbrain dopaminergic neurons controlled by the engrailed genes. J Neurosci 21: 3126-3134.

31. Sinclair, S. R., Fawcett, J. W., and Dunnett, S. B. 1999. Dopamine cells in nigral grafts differentiate prior to implantation. Eur J Neurosci 11: 4341-4348. 
32. Smidt, M. P., van Schaick, H. S., Lanctot, C., Tremblay, J. J., Cox, J. J., van der Kleij, A. A., Wolterink, G., Drouin, J., and Burbach, J. P. 1997. A homeodomain gene Ptx3 has highly restricted brain expression in mesencephalic dopaminergic neurons. Proc Natl Acad Sci U S A 94: 1330513310.

33. Sommer, L., Ma, Q., and Anderson, D. J. 1996. neurogenins, a novel family of atonal-related bHLH transcription factors, are putative mammalian neuronal determination genes that reveal progenitor cell heterogeneity in the developing CNS and PNS. Mol Cell Neurosci 8: 221-241.

34. Specht, L. A., Pickel, V. M., Joh, T. H., and Reis, D. J. 1981. Lightmicroscopic immunocytochemical localization of tyrosine hydroxylase in prenatal rat brain. I. Early ontogeny. J Comp Neurol 199: 233-253.

35. Sun, Y., Nadal-Vicens, M., Misono, S., Lin, M. Z., Zubiaga, A., Hua, X., Fan, G., and Greenberg, M. E. 2001. Neurogenin promotes neurogenesis and inhibits glial differentiation by independent mechanisms. Cell 104: 365-376.

36. Toresson, H., Mata de Urquiza, A., Fagerstrom, C., Perlmann, T., and Campbell, K. 1999. Retinoids are produced by glia in the lateral ganglionic eminence and regulate striatal neuron differentiation. Development 126: 13171326.

37. Wallen, A., Zetterstrom, R. H., Solomin, L., Arvidsson, M., Olson, L., and Perlmann, T. 1999. Fate of mesencephalic AHD2-expressing dopamine progenitor cells in NURR1 mutant mice. Exp Cell Res 253: 737-746.

38. Winkler, C., Kirik, D., and Bjorklund, A. 2005. Cell transplantation in Parkinson's disease: how can we make it work? Trends Neurosci 28: 86-92.

39. Witta, J., Baffi, J. S., Palkovits, M., Mezey, E., Castillo, S. O., and Nikodem, V. M. 2000. Nigrostriatal innervation is preserved in Nurr1-null mice, although dopaminergic neuron precursors are arrested from terminal differentiation. Brain Res Mol Brain Res 84: 67-78.

40. Zetterstrom, R. H., Solomin, L., Jansson, L., Hoffer, B. J., Olson, L., and Perlmann, T. 1997. Dopamine neuron agenesis in Nurr1-deficient mice. Science 276: 248-250. 